\title{
Ultra-relativistic heavy-ion collisions - a hot cocktail of hydrody- namics, resonances and jets
}

\author{
E. Zabrodin ${ }^{1,2, a}$, L. Bravina1 ${ }^{1}$, B.H. Brusheim Johansson ${ }^{1}$, J. Crkovska ${ }^{1,3}$, G.Kh. Eyyubova ${ }^{2,3}$,
} V.L. Korotkikh², I.P. Lokhtin ${ }^{2}$, L.V. Malinina ${ }^{2,4}$, S.V. Petrushanko ${ }^{2}$, and A.M. Snigirev ${ }^{2}$

${ }^{1}$ Department of Physics, University of Oslo, PB 1048 Blindern, Oslo, Norway

${ }^{2}$ Skobeltsyn Institute of Nuclear Physics, Moscow State University, RU-119991 Moscow, Russia

${ }^{3}$ Czech Technical University in Prague, FNSPE, Prague 110 00, Czech Republic

${ }^{4}$ Joint Institute for Nuclear Researches, Dubna, Russia

\begin{abstract}
Ultra-relativistic heavy-ion collisions at energies of RHIC and LHC are considered. For comparison with data the HYDJET++ model, which contains the treatment of both soft and hard processes, is employed. The study focuses mainly on the interplay of ideal hydrodynamics, final state interactions and jets, and its influence on the development of harmonics of the anisotropic flow. It is shown that jets are responsible for violation of the number-of-constituent-quark (NCQ) scaling at LHC energies. The interplay between elliptic and triangular flows and their contribution to higher flow harmonics and dihadron angular correlations, including ridge, is also discussed.
\end{abstract}

\section{Introduction}

Heavy-ion collisions at ultra-relativistic energies, accessible for modern colliders such as Relativistic Heavy Ion Collider (RHIC) at BNL and Large Hadron Collider (LHC) at CERN, revealed several signals which favored the creation of a new state of matter - quark-gluon plasma (QGP) - during the earlier phase of the collision. Among these signals are strong collective flow of secondary hadrons, particularly elliptic flow [1], and the phenomenon of jet quenching [2]. Recall, that the flow analysis in the azimuthal plane is performed in terms of Fourier series [3, 4]

$$
E \frac{d^{3} N}{d^{3} p}=\frac{1}{\pi} \frac{d^{2} N}{d p_{T}^{2} d y}\left\{1+2 \sum_{n=1}^{\infty} v_{n} \cos \left[n\left(\phi-\Psi_{n}\right)\right]\right\},
$$

where $\Psi_{n}$ is the azimuth of the participant plane of $n$-th order, $\phi$ is the azimuthal angle between the particle transverse momentum $p_{T}$ and the participant plane, and $y$ is the particle rapidity. The flow harmonic coefficients are

$$
v_{n}=\left\langle\cos \left[\left(n\left(\phi-\Psi_{n}\right)\right]\right\rangle,\right.
$$

where one has to average over all particles in a single event and then over all events, respectively. Harmonics are called directed, $v_{1}$, elliptic, $v_{2}$, triangular, $v_{3}$, quadrangular (sometimes, hexadecapole),

\footnotetext{
a e-mail: eugen.zabrodin@fys.uio.no
} 
$v_{4}$, pentagonal, $v_{5}$, hexagonal, $v_{6}$, flow and so on. The first two flow components have been studied in heavy-ion collisions at different energies for more than 15 years, whereas systematic investigation of triangular flow and higher harmonics started about 5 years ago. Although elliptic flow is the strongest among the flow harmonics, $v_{3}$ and higher order coefficients can carry valuable information about the properties of hot and dense medium at the very early stages of the fireball expansion. At energies of RHIC and LHC hydrodynamic models usually provide a good description of the $v_{2}\left(p_{T}\right)$ distribution at $p_{T} \leq 2 \mathrm{GeV} / c$. At higher transverse momenta the signal is overpredicted in hydrodynamics [5] in contrast to microscopic transport models, which underpredict the elliptic flow at high $p_{T}$ [6-8]. This gives rise to appearance of hybrid models, e.g. VISHNU [9] and MUSIC [10], where the hydrodynamic description of the early stage is coupled to hadronic cascade as afterburner after the chemical freeze-out.

Hard processes are usually not included in hydrodynamic models. In the flow sector it is just assumed that jets contribute to the $p_{T}$-distributions of the flow harmonics at high transverse momenta because of the jet quenching. Also, many hydrodynamic models rely on rather short tables of hadron resonances. Our aim is to show that resonances and jets are quite important for the description of not only the particle yields and $p_{T}$-spectra, but also collective effects such as anisotropic flow. The HYDJET++ model (HYDrodynamics with JETs) is employed for our study. Basic features of the model are listed in Sec. 2.

\section{Basic features of HYDJET++ model}

Monte Carlo event generator HYDJET++ [11] is the first model designed for the simulation of relativistic heavy-ion collisions which contains a hydrodynamics coupled to a hard multiparton state. Both soft and hard states are treated independently. The predecessors of the HYDJET++ in soft and hard sectors are FASTMC [12, 13] and PYQUEN [14] event generators, respectively. The soft part of the model represents a relativistic hydrodynamical parametrization of the chemical (single freezeout scenario) or thermal (double freeze-out scenario) freeze-out hypersurfaces with given freeze-out conditions. The simulation of an individual event starts with calculation of effective volume $V_{\text {eff }}$ of the fireball. This volume depends on the mean number of participating nucleons at given centrality, i.e. impact parameter $b$, of the collision. The latter is determined from the Glauber model of multiple scattering. For the most appropriate scheme with separated chemical and thermal freeze-out, the particle composition in the system is frozen at the stage of chemical freeze-out. The fireball continues to expand and cools down until the thermal freeze-out stage, where the contact between hadrons is lost. The final state interactions (FSI) take into account the two- and three-body decays of the resonances. The model benefits from the extremely rich table of resonances with ca. 350 particles taken from the SHARE thermal model [15]. Note, that HYDJET++ employs its own original routine for treatment of resonance decays.

In hard sector the model propagates the hard partons through the expanding quark-gluon plasma and takes into account both gluon radiation loss and collisional loss because of the parton rescattering. For each hard nucleon-nucleon $(\mathrm{NN})$ collision the PYQUEN routine starts with generation of initial parton spectra and production vertexes at a given impact parameter. In recent version of the model $[16,17]$ the tune Pro-Q20 of PYTHIA is utilized, whereas earlier modifications $[14,18]$ relied on standard PYTHIA_6.4 [19]. After the rescattering stage accompanied by radiative and collisional energy loss the partons and in-medium emitted gluons are hadronized according to the Lund string model. The hard event includes also jets. Their number is proportional to the product of number of binary $\mathrm{NN}$ collisions in an event at given impact parameter and the integral cross section of the hard processes in NN collision with the minimal transverse momentum transfer, $p_{T}^{\min }$. 
The flow components are implemented in the HYDJET++ as follows. For noncentral collisions the transverse radius of the overlap region is a function of impact parameter $b$, azimuthal angle $\phi$ and spatial eccentricity $\epsilon(b)=\left(R_{y}^{2}-R_{x}^{2}\right) /\left(R_{y}^{2}+R_{x}^{2}\right)[13]$,

$$
R_{\text {ell }}(b, \phi)=R_{\text {fr. }- \text { out }}(b) \sqrt{\frac{1-\epsilon^{2}(b)}{1+\epsilon(b) \cos 2 \phi}}
$$

with

$$
R_{f r .- \text { out }}(b)=R_{0} \sqrt{1-\epsilon(b)}
$$

In the last formula $R_{0} \equiv R_{f r-\text { out }}(0)$ is the freeze-out radius of the fireball in a central collision. The momentum anisotropy arises from the pressure gradients, which are stronger in the direction of short axis of the ellipsoid. Then, the azimuthal angle $\phi_{f l}$ in HYDJET++ does not coincide with the azimuthal angle $\phi$ as in the case of transverse isotropic parametrization. Instead, both angles are linked via the nonzero flow anisotropy parameter $\delta(b)$ as [13]

$$
\tan \phi_{f l}=\sqrt{\frac{1-\delta(b)}{1+\delta(b)}} \tan \phi
$$

Parameters $\epsilon(b)$ and $\delta(b)$ are proportional to the initial spatial anisotropy $\epsilon_{0}=b /\left(2 R_{A}\right)$.

Triangular flow in the model is obtained by further modification of the transverse radius [16]

$$
R_{\text {triang }}(b, \phi)=R_{\text {ell }}(b, \phi)\left\{1+\epsilon_{3}(b) \cos \left[3\left(\phi-\Psi_{3}\right)\right]\right\} .
$$

Experimental data indicate no correlations between the reaction planes $\Psi_{2}$ and $\Psi_{3}$, therefore position of the plane $\Psi_{3}$ in the generated events is isotropically distributed w.r.t. the plane $\Psi_{2} . \epsilon_{3}(b)$ is a new free parameter responsible for appearance of triangularity in the system. It can be treated independently or expressed via the initial ellipticity $\epsilon_{0}(b)$. It is worth mentioning that introduction of triangular modulation in the system does not change the elliptic flow $v_{2}$. Further details of the model can be found elsewhere [11-14]. Here we would like to stress that the model extension to triangular flow appears very fruitful. It enables one to study the cross-talk of elliptic and triangular harmonics and their contributions to higher flow harmonics in ideal conditions, because all other eccentricities, such as $\epsilon_{4}, \epsilon_{5}$, etc., are absent. Several important consequences of the interplay between $v_{2}$ and $v_{3}$ are discussed below.

\section{Elliptic flow. Interplay of hydrodynamics and jets}

Elliptic flow of hadrons in $\mathrm{Au}+\mathrm{Au}$ collisions at RHIC and in $\mathrm{Pb}+\mathrm{Pb}$ collisions at $\mathrm{LHC}$ was among the first collective effects considered within the HYDJET++ model. First of all, it appeared that the differential flow $v_{2}\left(p_{\mathrm{T}}\right)$ had a characteristic hump structure around $2.5-3.5 \mathrm{GeV} / c$, see Fig. 1, where the elliptic flow reached its maximum value and then rapidly dropped. In experimental data such a hump profile became pronounced when the methods based on particle cumulants and Lee-Yang zeroes (LYZ) were extended to the range of intermediate transverse momenta $2 \mathrm{GeV} / c \leq p_{\mathrm{T}} \leq 5 \mathrm{GeV} / c$. Standard two-particle correlation method gives almost flat distribution of $v_{2}\left(p_{\mathrm{T}}\right.$ at $p_{\mathrm{T}} \geq 2.5 \mathrm{GeV} / c$. Then, the model demonstrated crossing of meson and baryon branches, as shown in Fig. 1. Here the elliptic flows of identified charged hadrons are compared to those measured by PHENIX and STAR collaborations in Au+Au collisions at full RHIC energy $\sqrt{s}=200 \mathrm{AGeV}$. Note, that in ideal hydrodynamics the $v_{2}\left(p_{\mathrm{T}}\right)$ distributions of hadrons grow without crossing up to unity. Both effects, i.e. 


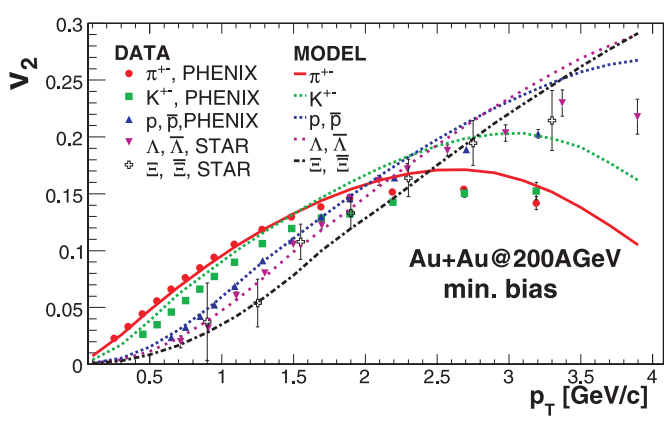

Figure 1. The $v_{2}\left(p_{T}\right)$ distributions in the HYDJET++ model for different hadron species (lines) and comparison with RHIC data (symbols) (from [21]).

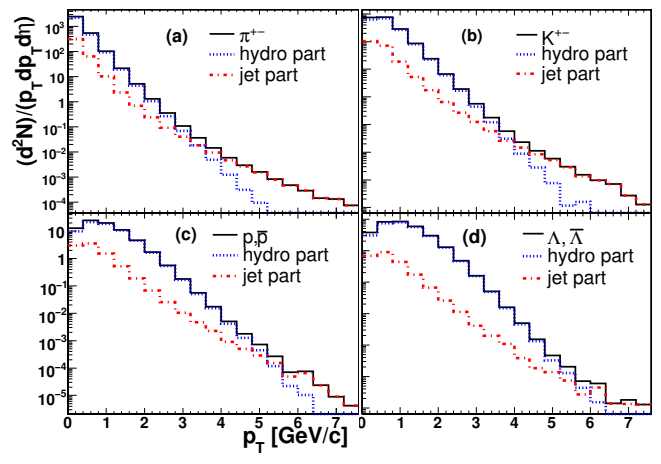

Figure 2. The $p_{T}$ distribution for charged pions, kaons, protons and antiprotons, and Lambdas in HYDJET++ calculations of $\mathrm{Pb}+\mathrm{Pb}$ collisions with centrality $c \approx$ $40 \%$ at LHC (from [20]).

(1) the saturation and the subsequent drop of hadron elliptic flow with rising transverse momentum, and (2) the crossing of meson and baryon branches demonstrate clearly the influence of jets. Figure 2 presents the $p_{\mathrm{T}}$ spectra of most abundant particle species in comparison to their hydrodynamic (soft) and jet (hard) parts. Lighter hadrons have softer $p_{\mathrm{T}}$-spectra, thus the slopes of their hydrodynamically generated distributions are steeper. Therefore, the change from hydro-dominated to jet-dominated regime occurs for mesons at lower $p_{\mathrm{T}}$ compared to that for baryons, as was pointed out in [20, 21]. Hadrons emerging from the jet fragmentation develop very weak elliptic flow due to well-known jet quenching process, and the combined $v_{2}\left(p_{\mathrm{T}}\right)$ drops at certain $p_{\mathrm{T}}$ threshold. At higher transverse momenta heavier hadrons possess larger $v_{2}$.

Another interesting feature of elliptic flow which was considered as a "smoking gun" revealing the QGP formation is the number-of-constituent-quark (NCQ) scaling, first observed in gold-gold collisions at RHIC in $[22,23]$. If the elliptic flow $v_{2}$ and the transverse kinetic energy $K E_{\mathrm{T}}=m_{\mathrm{T}}-m_{0}$ are divided by a number of constituent quarks $n_{q}, 2$ for mesons and 3 for baryons, then the distributions $v_{2} / n_{q}\left(K E_{\mathrm{T}} / n_{q}\right)$ for all hadrons coincide with good accuracy up to $K E_{\mathrm{T}} / n_{q} \approx 1 \mathrm{GeV}$. This circumstance was taken as a strong evidence of the predominant production of elliptic flow from the quark coalescence at the partonic, i.e. plasma, stage. However, in $\mathrm{Pb}+\mathrm{Pb}$ collisions at $\mathrm{LHC}$ energy $\sqrt{s}=2.76 \mathrm{ATeV}$ the NCQ scaling was found to be violated [24], whereas recent results of beam energy scan (BES) at RHIC confirm the scaling fulfillment in a broad energy range $7.7 \mathrm{AGeV} \leq \sqrt{s} \leq$ $62 \mathrm{AGeV}$ [25]. At the first sight the results look puzzling, although the violation of NCQ scaling at LHC energies was predicted in [20]. Jet phenomena are again the processes which should be responsible for worsening of the scaling. Figure 3 displays the reduced functions $v_{2}\left(K E_{\mathrm{T}} / n_{q}\right)$ calculated within the HYDJET++ model for $20 \%-30 \%$ central $\mathrm{Pb}+\mathrm{Pb}$ collisions at $2.76 \mathrm{ATeV}$. The panels show the elliptic flow of only directly produced hadrons, the flow modified by decays of resonances (jet contribution is excluded), and the final $v_{2}$ of hadrons, where both hard and soft processes are taken into account. One can see in Fig. 3 that resonances are extremely important, because they drive the particle elliptic flow towards the NCQ scaling. To see the degree of the scaling fulfillment explicitly all particle flows in the bottom row are normalized to the flow of protons, $v_{2}^{h} / n_{q}: v_{2}^{p} / n_{q}$. The feeddown from heavy resonances makes the elliptic flow of light hadrons harder, whereas, for instance, flow of $\phi$ mesons is unchanged due to the absence of the resonance boost. But then jets come into 


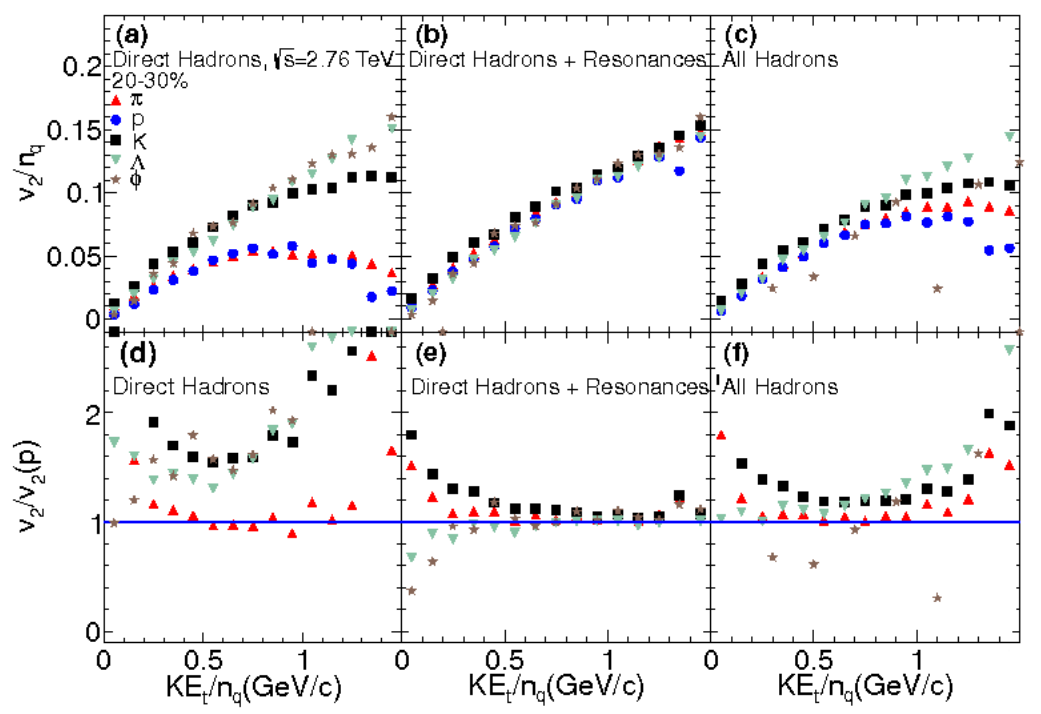

Figure 3. Upper row: The $K E_{T} / n_{q}$ dependence of elliptic flow for (a) direct hadrons, (b) hadrons produced both directly and from resonance decays, and (c) all hadrons produced in the HYDJET++ model for $\mathrm{Au}+\mathrm{Au}$ collisions at $\sqrt{s}=200 \mathrm{~A} \mathrm{GeV}$ with centrality $20--30 \%$. Bottom row: The $K E_{T} / n_{q}$ dependence of the ratios $\left(v_{2} / n_{q}\right) /\left(v_{2}^{p} / 3\right)$ for (d) direct hadrons, (e) direct hadrons plus hadrons from the decays, and (f) all hadrons (from [26]).

play and spoil the result completely. In heavy-ion collisions jets are more abundant at LHC energies than at RHIC ones, therefore, the NCQ scaling at LHC holds only approximately despite the fact that in pure hydrodynamic sector with final state interactions the scaling performance is very good.

Figure 4 demonstrates the importance of final state interactions for the development of hadron elliptic flow. Only $v_{2}$ of kaons seems to be unchanged, while the flows of charged pions, protons + antiprotons and Lambdas become stronger. Soft pions coming from the resonance decays possess lower momentum anisotropy. On the other hand, hard pions and heavier particles have larger elliptic flow attributed to massive resonances.

\section{Triangular flow}

Triangular flow $v_{3}$ was introduced in the HYDJET++ model relatively recently [16, 17]. Figure 5 shows the model generated $v_{3}$ of charged hadrons in $\mathrm{Pb}+\mathrm{Pb}$ collisions at $2.76 \mathrm{ATeV}$ compared to CMS data [27]. In order to estimate uncertainties in experimental determination of the $v_{3}$ signal, the eventplane (EP) method was applied to restore the triangular flow out of the generated particle spectra. The restored triangular flow is plotted in Fig. 5 also. One can see that overall agreement with the data is good. At transverse momenta below $3 \mathrm{GeV} / c$ the "true" flow $v_{3}\left(\Psi_{3}^{\mathrm{RP}}\right)$ coincides with that restored by the event-plane method, $v_{3}\{E P\}$. At $3 \mathrm{GeV} / c \leq p_{T} \leq 4 \mathrm{GeV} / c v_{3}\{E P\}$ exceeds slightly the original flow. Both restored and generated differential triangular flows show a falloff at $p_{T} \geq 3.5 \mathrm{GeV} / c$ in accord with the data. As in the case with elliptic flow, such a drop in the flow behavior is caused by hadrons originated from the fragmentation of jets. These hadrons, which carry very little collective 


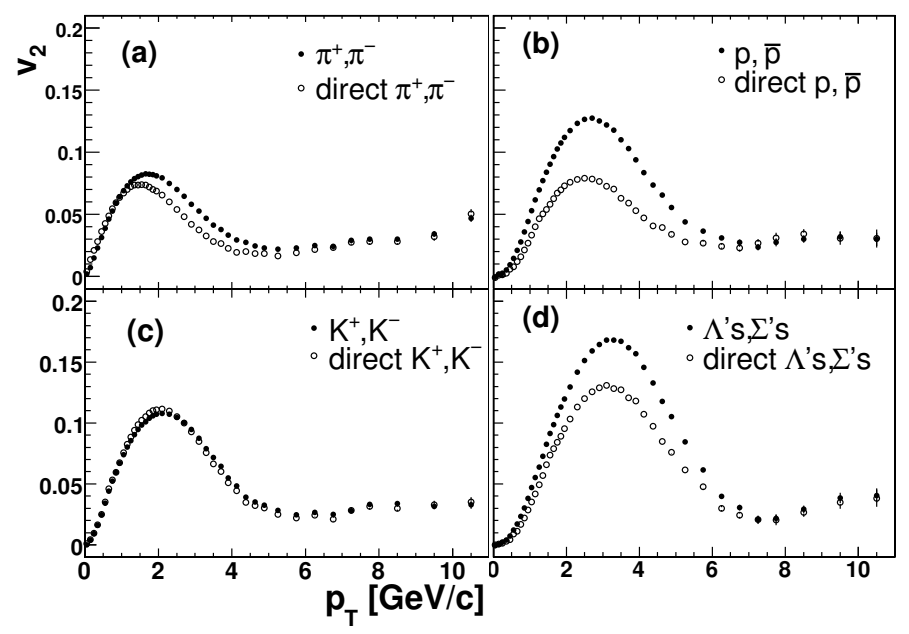

Figure 4. Transverse momentum dependence of elliptic flow of direct hadrons (open symbols) and of all hadrons (full symbols) produced in the HYDJET ++ model for $\mathrm{Pb}+\mathrm{Pb}$ collisions at LHC energy with centrality $c \approx 40 \%$ : (a) protons, (b) pions, (c) kaons, and (d) Lambdas plus Sigmas (from [20]).
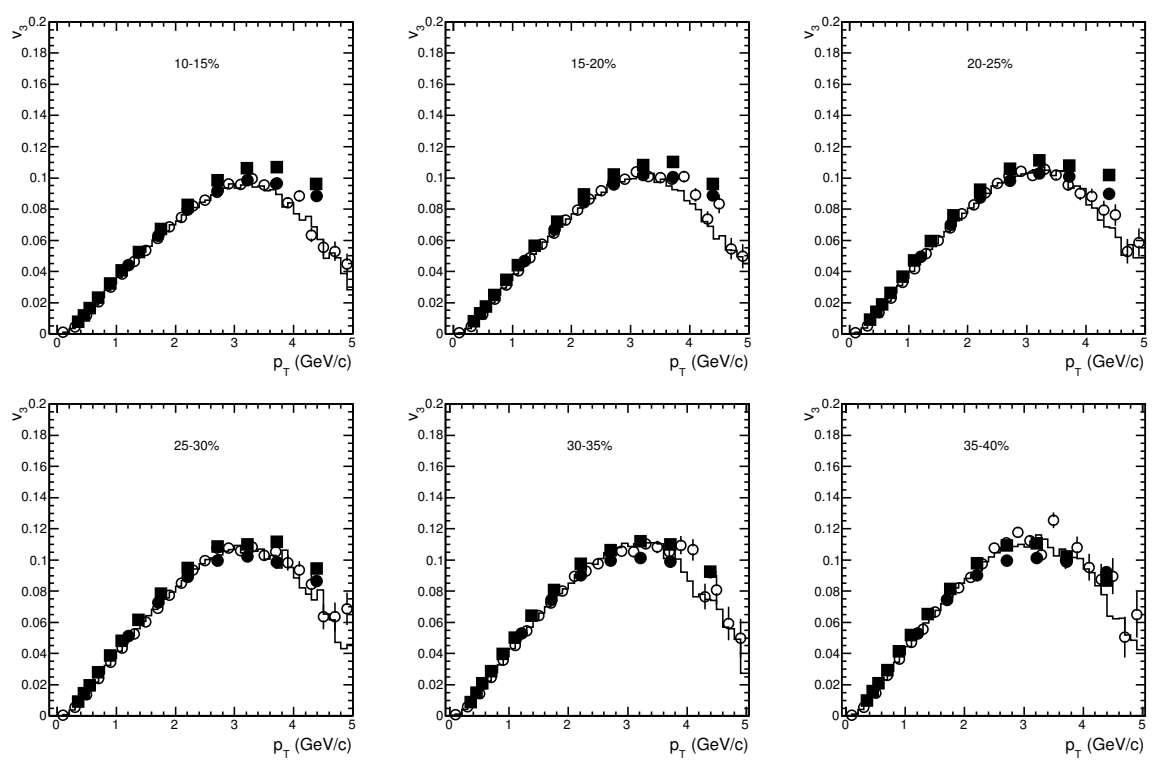

Figure 5. Transverse momentum dependence of triangular flow of charged hadrons in pseudorapidity interval $|\eta|<0.8$ for six different centralities of $\mathrm{Pb}+\mathrm{Pb}$ collisions at $\sqrt{s}=2.76 \mathrm{TeV}$. The full symbols are CMS data [27] $\left(v_{3}\{2\}\right.$ - circles, $v_{3}\{\mathrm{EP}\}-$ squares $)$, open circles and histograms represent $v_{3}\{\mathrm{EP}\}$ and $v_{3}\left(\Psi_{3}^{\mathrm{RP}}\right)$ for HYDJET ++ events, respectively (from [16]). 

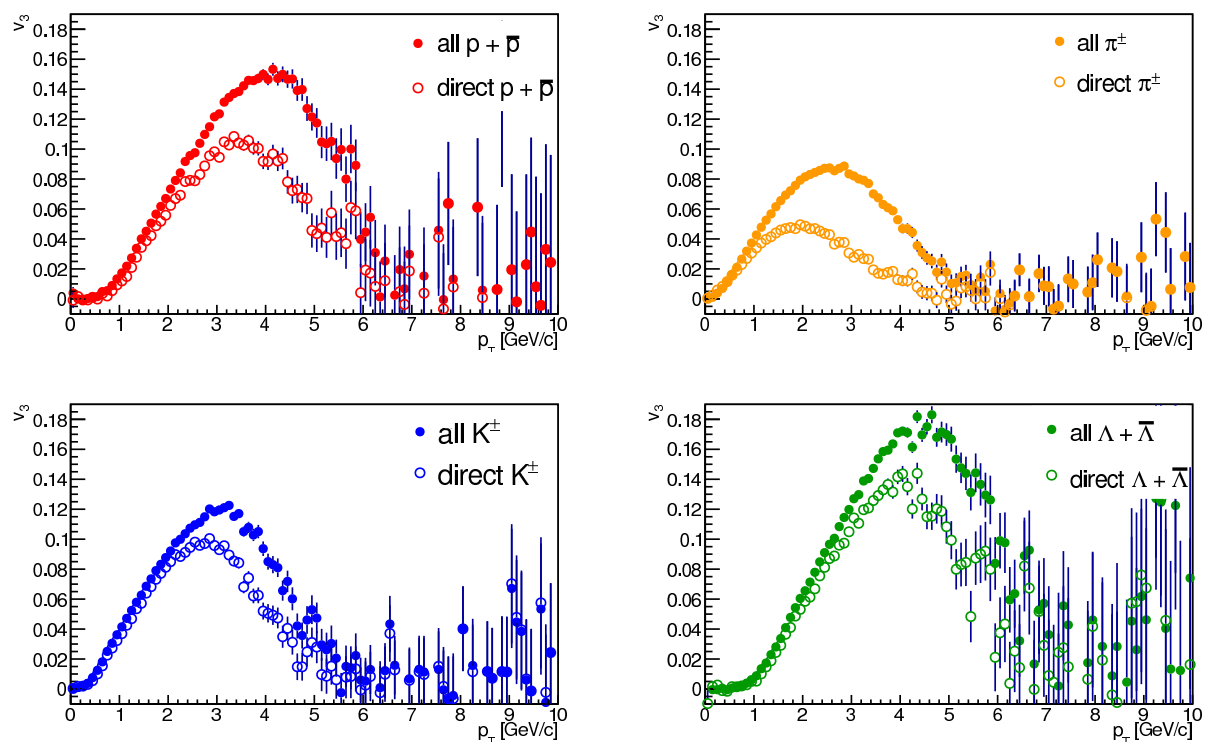

Figure 6. Triangular flow vs. $p_{T}$ of direct hadrons (open symbols) and of all hadrons (full symbols) in HYD$\mathrm{JET}++$ calculations of $20-30 \%$ central $\mathrm{Pb}+\mathrm{Pb}$ collisions at $\sqrt{s}=2.76 \mathrm{TeV}$.

flow, if any, dominate the particle spectrum at intermediate and high $p_{T}$. Generally speaking, there are many similarities between the role of jets and final state interactions, i.e. resonance decays, in the development of elliptic and triangular flows. For instance, $v_{3}\left(p_{T}\right)$ after the decays of resonances is increased and its maximum is shifted to higher transverse momenta, as seen in Fig. 6. The counterplay of jets and resonances also leads to violation of NCQ scaling conditions for triangular flow. Details can be found in J. Crkovska's talk [28] (this volume).

\section{Higher flow harmonics}

Systematic study of higher flow harmonics $v_{n}, n>3$ started relatively recently. Important theoretical result was obtained in [29], where the authors demonstrated that the quadrangular flow $v_{4}$ is determined exclusively by the elliptic flow $v_{2}$

$$
v_{4} \simeq \frac{1}{2} v_{2}^{2}
$$

at high transverse momenta. Since triangular flow does not affect the $v_{4}$, here we will consider pentagonal and hexagonal flows, respectively. Study of the fulfillment of Eq.(7) in the model and the role of jets in the increase of the ratio $v_{4} / v_{2}^{2}$ and rise of its high- $p_{T}$ tail can be found in $[26,30,31]$.

Pentagonal $v_{5}$ and hexagonal $v_{6}$ flow coefficients in non-central lead-lead collisions at $\sqrt{s}=$ $2.76 \mathrm{TeV}$ are shown as functions of $p_{T}$ in Figs. 7 and 8, respectively. Here the HYDJET++ results are compared with CMS data, comparison with ATLAS data can be found in [16]. Because of absence of genuine eccentricity $\epsilon_{5}$ and related to it reaction plane $\Psi_{5}$ in the present version of the model, the generated $v_{5}$ shown in Fig. 7 emerges only as a result cross-talk of $v_{2}$ and $v_{3}$, namely $v_{5} \sim v_{2} \cdot v_{3}$ [32]. Direct check in HYDJET++ reveals that $v_{5}=0$ if either elliptic or triangular flow is absent. If, 
EPJ Web of Conferences
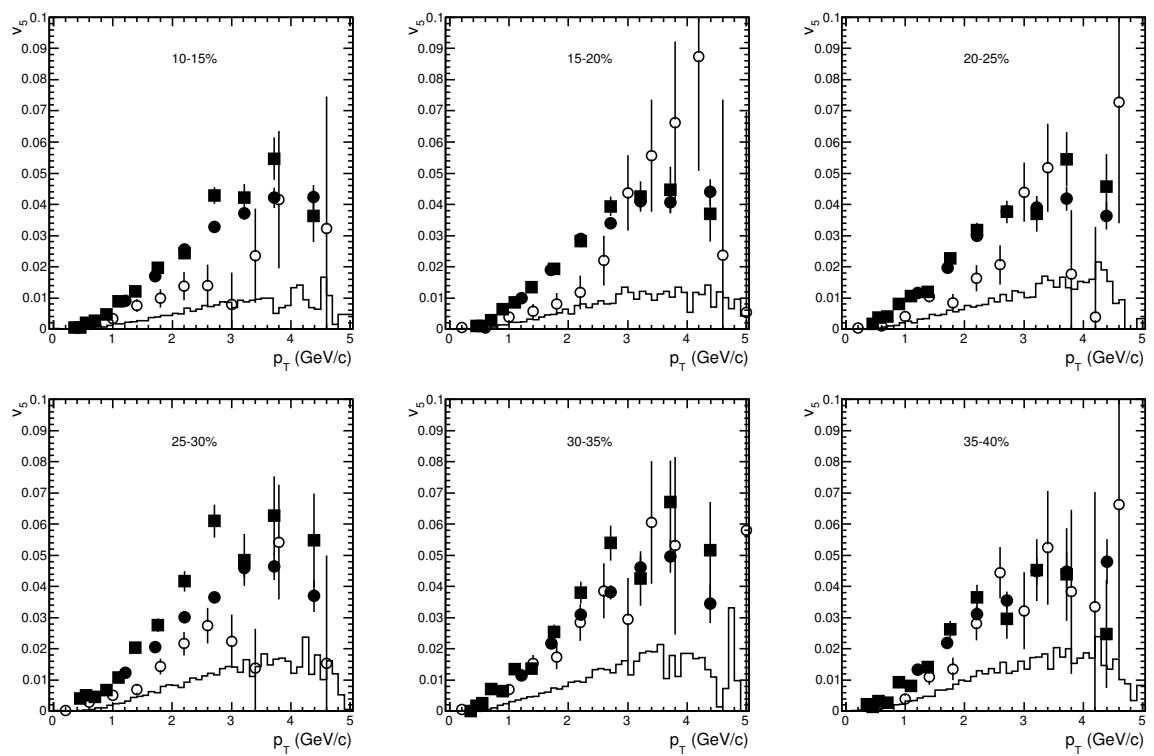

Figure 7. The same as 5 but for pentagonal flow of charged hadrons in $\mathrm{Pb}+\mathrm{Pb}$ collisions at $\sqrt{s}=2.76 \mathrm{TeV}$ (from [16]).
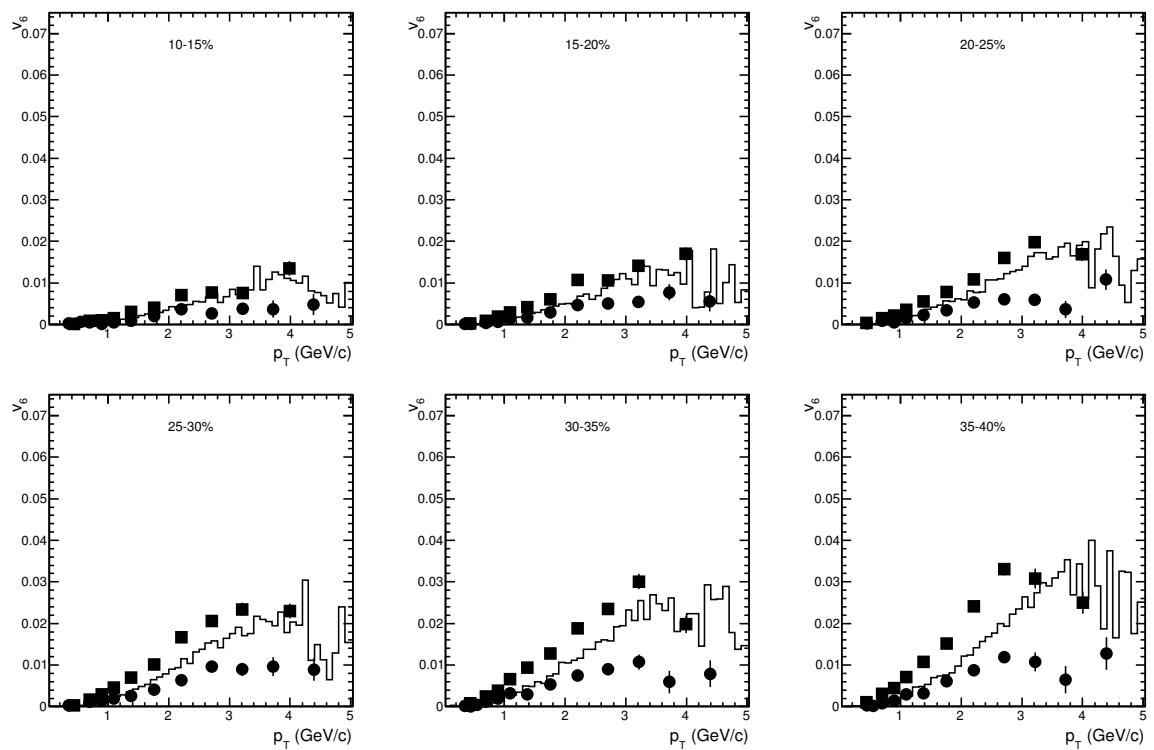

Figure 8. The same as 7 but for hexagonal flow of charged hadrons in $\mathrm{Pb}+\mathrm{Pb}$ collisions at $\sqrt{s}=2.76 \mathrm{TeV}$ (from [16]). 


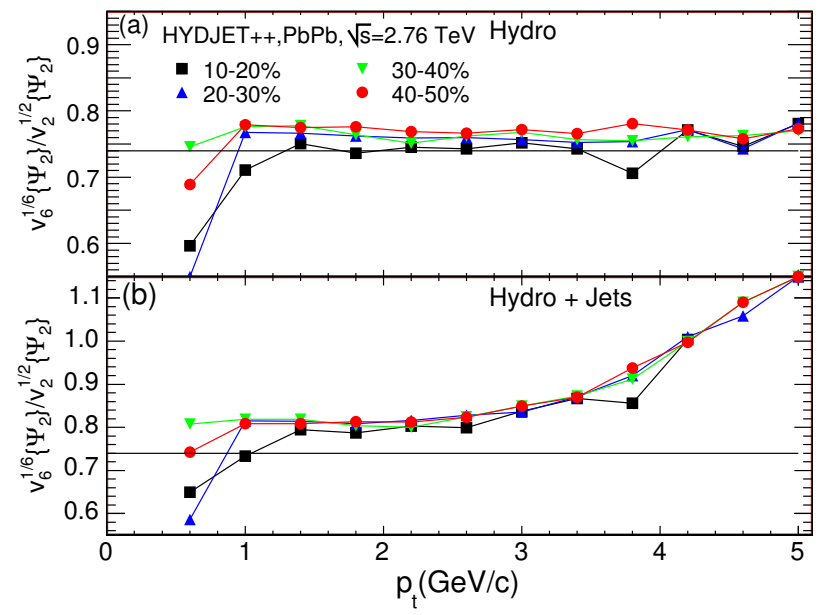

Figure 9. Ratio $v_{6}^{1 / 6} / v_{2}^{1 / 2}$ as a function of $p_{T}$ in the $\Psi_{2}$ event plane for charged particles, originated from (a) soft processes only and (b) both soft and hard processes, in HYDJET ++ simulations of $\mathrm{Pb}+\mathrm{Pb}$ collisions at $\sqrt{s}=2.76 \mathrm{TeV}$ for four selected centralities. Solid lines in both plots show the prediction of ideal hydrodynamics for this ratio at high $p_{t}, v_{6}^{1 / 6} / v_{2}^{1 / 2}=(1 / 6)^{1 / 6} \approx 0.74$ (from [17]).

however, the event-plane method is applied to generated particle spectra, the reconstructed pentagonal flow in the model appears to be very close to the distributions extracted from the experimental data, especially for semi-peripheral events.

The situation with the hexagonal flow is even more curious, because the $v_{6}$ emerging in the model is not a product but rather a sum of independent contributions coming from $v_{2}$ and $v_{3}$. Within the same approach as was used for derivation of Eq.(7), it was obtained that

$$
v_{6} \simeq \frac{1}{6} v_{2}^{3}+\frac{1}{2} v_{3}^{2}
$$

in the high- $p_{T}$ limit [17]. This leads to a non-trivial correlations between the $\left(\Psi_{2}, \Psi_{6}\right)$ and $\left(\Psi_{3}, \Psi_{6}\right)$ event planes. Triangular flow weakly increases with rising non-centrality of the collisions, whereas elliptic flow is almost zero for central 0-5\% collisions and quickly rises up as the reactions become more peripheral. As a result, for central events hexagonal flow is mainly determined by the $v_{3}$, and $\Psi_{6}$ plane should be closer to $\Psi_{3}$ plane. In semi-peripheral and peripheral collisions the situation is opposite. Here elliptic flow dominates over the triangular flow, thus $\Psi_{6}$ is elongated closer to $\Psi_{2}$ rather than to $\Psi_{3}$ [17]. Such a behavior was observed in plane correlators $\left\langle\cos 6\left(\Psi_{2}-\Psi_{6}\right)\right\rangle$ and $\left\langle\cos 6\left(\Psi_{3}-\Psi_{6}\right)\right\rangle$ in [33]. Another interesting feature is the prediction of the scaling for $v_{6}^{1 / 6}\left\{\Psi_{2}\right\} / v_{2}^{1 / 2}\left\{\Psi_{2}\right\}$ ratios, where the $p_{T}$-distributions for different centralities should sit on the top of each other [17], as displayed in Fig.9. In contrast, for the similar ratios $v_{6}^{1 / 6}\left\{\Psi_{3}\right\} / v_{3}^{1 / 3}\left\{\Psi_{3}\right\}$ the scaling is not observed. These predictions can be easily checked experimentally. Again, jets increase the ratio of about $10 \%$ and cause the rise of the high- $p_{T}$ tail at $p_{T} \geq 3 \mathrm{GeV} / c$. Other details concerning the peculiarities of the hexagonal flow development in HYDJET ++ can be found in [16, 17, 31, 34]. 


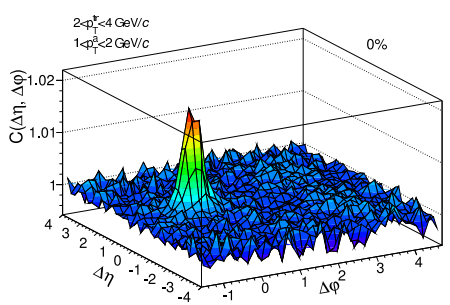

(a)

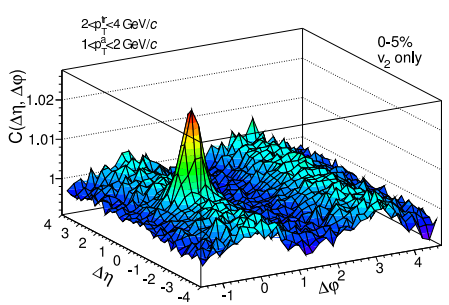

(b)

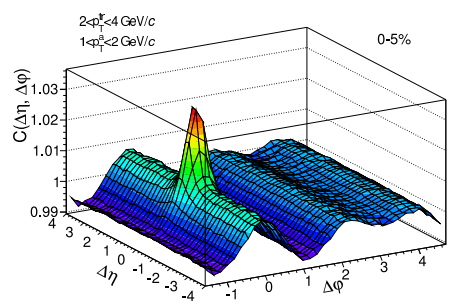

(c)

Figure 10. 2D correlation function in HYDJET ++ in $\mathrm{Pb}+\mathrm{Pb}$ collisions at $\sqrt{s_{\mathrm{NN}}}=2.76 \mathrm{TeV}$ for $2<p_{\mathrm{T}}^{\mathrm{tr}}<4$ $\mathrm{GeV} / c$ and $1<p_{\mathrm{T}}^{\mathrm{a}}<2 \mathrm{GeV} / c$ for (a) central collisions with impact parameter $b=0$, (b) centrality $0-5 \%$ with only elliptic flow, and (c) centrality $0-5 \%$ with both elliptic and triangular flow present (from [38]).

\section{Dihadron angular correlations: ridge}

The two-particle correlations in the system may arise because of various interaction processes between particles and phase-space restrictions imposed by, e.g. energy-momentum conservation. The twoparticle correlation function is typically defined as the ratio of pair distribution in the event to the combinatorial background of uncorrelated particles. In the flow dominated regime the pair angular distribution reads [cf. Eq.(1)]

$$
\frac{d N^{\text {pairs }}}{d \Delta \varphi} \propto 1+2 \sum_{n=1}^{\infty} V_{n}\left(p_{\mathrm{T}}^{\mathrm{tr}}, p_{\mathrm{T}}^{\mathrm{a}}\right) \cos n(\Delta \varphi),
$$

where $\Delta \varphi=\varphi^{\mathrm{tr}}-\varphi^{\mathrm{a}}$, and indices "tr" and "a" indicate the so-called "trigger" and "associated" particle, respectively. The study of angular dihadron correlations in relativistic heavy-ion collisions revealed the long-range correlations dubbed "ridge" [35]. Many interesting options have been proposed for the description of the ridge phenomenon, e.g. Cerenkov gluon radiation, Mach-cone of shock waves, etc. (see [36] and references herein). The authors of [37] suggested that the triangular flow should be important for understanding of ridge. HYDJET++ is ideally suited for such a check. - Although the model possesses many sources of particle correlations, for instance decays of resonances, femtoscopic correlations, jets, the long-range correlations in the model appear only because of the collective flow. The correlation function $C(\Delta \eta, \Delta \varphi)$ calculated in HYDJET ++ for central lead-lead collisions at $\sqrt{s}=$ $2.76 \mathrm{TeV}$ is shown in Fig.10(a)-(c). In Fig.10(a) the centrality is $0 \%$ sharp, and both elliptic and triangular flows are absent. Just the distinct near-side $(\Delta \varphi \approx 0)$ jet peak is seen, and no long range azimuthal correlations at the near-side or away-side $(\Delta \varphi \approx \pi)$ are found. In Fig.10(b) the centrality is $0-5 \%$, but the triangular flow is switched off. Here the long range correlations start to appear at both sides. However, only the presence of triangular flow in addition to the elliptic one [see Fig.10(c)] leads to development of ridge at near-side and, simultaneously, to formation of characteristic double-hump structure at the away-side, in full agreement with the experimental observations. Details of the study are presented in [38].

\section{Conclusions}

We show the importance of final state interactions and jets for the correct treatment of anisotropic flow harmonics in heavy-ion collisions at ultrarelativistic energies within the ideal hydrodynamic model. Jets account for (i) falloff of the flow harmonics in the range of intermediate transverse momenta, (ii) 
changing of the mass ordering of the hadron elliptic flow, (iii) increase of the ratios $v_{n}^{1 / n}\left(p_{T}\right) / v_{2}^{1 / 2}\left(p_{T}\right)$ of about $10-15 \%$ and formation of rising high- $p_{T}$ tails in these ratios. Maybe, the most important result is that (iv) jets are responsible for the violation of the constituent quark scaling at energies of LHC and higher.

Decays of resonances (i) significantly enhance the elliptic and triangular flows of hadrons, (ii) make the $v_{n}\left(p_{T}\right)$-distributions harder and (iii) push the particle spectra towards the NCQ-scaling fulfillment. Also, the interplay of $v_{2}$ and $v_{3}$ is studied in almost ideal conditions. We demonstrate that significant part of higher flow harmonics $v_{4}, v_{5}, v_{6}$, etc. comes from the elliptic and triangular flows. Therefore, in order to investigate the genuine higher harmonics related to higher-order eccentricities one has to study more central events, where at least the elliptic flow is weak. Finally, the interplay between $v_{2}$ and $v_{3}$ is able to describe both qualitatively and quantitatively the long-range correlations, i.e. ridge at near-side and double-hump structure at the away-side. These phenomena deserve further investigations.

\section{Acknowledgments}

This work was supported in parts by the Department of Physics, UiO, Russian Foundation for Basic Research under Grant No. 12-02-91505, Grant of the President of Russian Federation for Scientific School Supporting No. 3042.2014.2, and European Social Fund within the framework of realizing the project Support of Inter-sectoral Mobility and Quality Enhancement of Research Teams at Czech Technical University in Prague, CZ.1.07/2.3.00/30.0034.

\section{References}

[1] J.-Y. Ollitrault, Phys. Rev. D 46, 229 (1992)

[2] X.-N. Wang and M. Gyulassy, Phys. Rev. Lett. 68, 1480 (1992)

[3] S. A. Voloshin and Y. Zhang, Z. Phys. C 70, 665 (1996)

[4] A. M. Poskanzer and S. A. Voloshin, Phys. Rev. C 58, 1671 (1998)

[5] S. A. Voloshin, A. M. Poskanzer, and R. Snellings, in Relativistic Heavy Ion Physics, LandoltBörnstein Database Vol. 23, edited by R. Stock (Springer, Berlin, 2010), p.5-54.

[6] M. Bleicher and H. Stöcker, Phys. Lett. B 526, 309 (2002).

[7] E. E. Zabrodin, C. Fuchs, L. V. Bravina, and Amand Faessler, Phys. Lett. B 508, 184 (2001).

[8] G. Burau et al., Phys. Rev. C 71, 054905 (2005).

[9] H. Song, S. A. Bass, and U. Heinz, Phys. Rev. C 83, 024912 (2011).

[10] B. Schenke, S. Jeon, and C. Gale, Phys. Rev. C 82, 014903 (2010).

[11] I. P. Lokhtin et al., Comput. Phys. Commun. 180, 779 (2009)

[12] N. S. Amelin et al., Phys. Rev. C 74, 064901 (2006)

[13] N. S. Amelin et al., Phys. Rev. C 77, 014903 (2008)

[14] I. P. Lokhtin and A. M. Snigirev, Eur. Phys. J. C 46, 211 (2006)

[15] G. Torrieri et al., Comput. Phys. Commun. 167, 229 (2005)

[16] L. V. Bravina et al., Eur. Phys. J. C 74, 2807 (2014)

[17] L. V. Bravina et al., Phys. Rev. C 89, 024909 (2014)

[18] I. P. Lokhtin et al., Eur. Phys. J. C 72, 2045 (2012)

[19] T. Sjostrand, S. Mrenna, and P. Skands, JHEP 0605, 026 (2006)

[20] G. Eyyubova et al., Phys. Rev. C 80, 064907 (2009)

[21] E. Zabrodin et al., J. Phys. G. 37, 094060 (2010) 
[22] J. Adams et al. (STAR Collaboration), Phys. Rev. Lett. 92, 052302 (2004).

[23] S. S. Adler et al. (PHENIX Collaboration), Phys. Rev. Lett. 91, 182301 (2003).

[24] F. Noferini et al. (ALICE Collaboration), Nucl. Phys. A 904-905, 438c (2013)

[25] L. Adamczyk et al. (STAR Collaboration), Phys. Rev. C 88, 014902 (2013)

[26] L. Bravina, B. H. Brusheim Johansson, G. Eyyubova, and E. Zabrodin, Phys. Rev. C 87, 034901 (2013)

[27] S. Chatrchyan, et al. (CMS Collaboration), Phys. Rev. C 87, 014902 (2013)

[28] J. Crkovska et al., (these Proceedings)

[29] N. Borghini and J.-Y. Ollitrault, Phys. Lett. B 642, 227 (2006)

[30] E. Zabrodin, G. Eyyubova, L. Malinina, and L. Bravina, Acta Phys. Polon. B 5, 349 (2012)

[31] B. H. Brusheim Johansson et al., (these Proceedings)

[32] D. Teaney and L. Yan, Phys. Rev. C 86, 044908 (2012)

[33] J. Jia et al. (ATLAS Collaboration), Nucl. Phys. A 910-911, 276 (2013)

[34] E. Zabrodin et al., EPJ Web Confer. 71, 00142 (2014)

[35] A. Adare et al. (PHENIX Collaboration), Phys. Rev. C 78, 014901 (2008);

M.M. Aggarwal et al. (STAR Collaboration), Phys. Rev. C 82, 024912 (2010);

K. Aamodt et al. (ALICE Collaboration), Phys. Rev. Lett. 107, 032301 (2011)

[36] Proceedings of Quark Matter 2008, J. Phys. G 35, 104001-104167 (2008)

[37] B. Alver and G. Roland, Phys. Rev. C 81, 054905 (2010) [Erratum-ibid. C 82, 039903 (2010)]

[38] G. Eyyubova et al., arXiv:1411.4487 [hep-ph] 